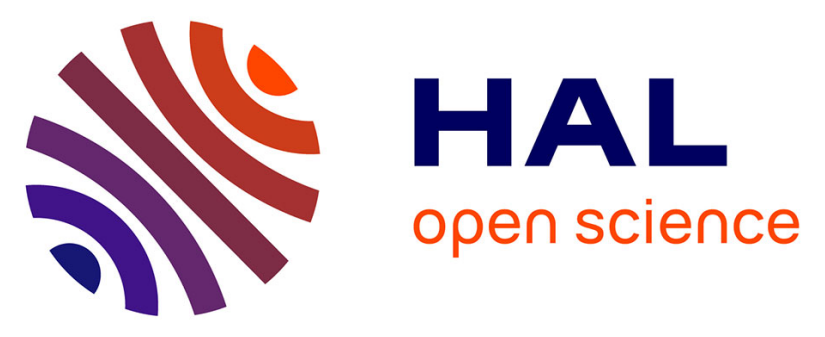

\title{
High-Frequency Mechanical Properties of Tumors Measured by Brillouin Light Scattering
}

Jeremie Margueritat, Angélique Virgone-Carlotta, Sylvain Monnier, Hélène

Delanoë-Ayari, Hichem C Mertani, Alice Berthelot, Quentin Martinet, Xavier

Dagany, Charlotte Rivière, Jean-Paul Rieu, et al.

\section{To cite this version:}

Jeremie Margueritat, Angélique Virgone-Carlotta, Sylvain Monnier, Hélène Delanoë-Ayari, Hichem C Mertani, et al.. High-Frequency Mechanical Properties of Tumors Measured by Brillouin Light Scattering. Physical Review Letters, 2019, 122 (1), pp.018101. 10.1103/PhysRevLett.122.018101. hal-02107154

\section{HAL Id: hal-02107154 \\ https://hal.science/hal-02107154}

Submitted on 22 Oct 2020

HAL is a multi-disciplinary open access archive for the deposit and dissemination of scientific research documents, whether they are published or not. The documents may come from teaching and research institutions in France or abroad, or from public or private research centers.
L'archive ouverte pluridisciplinaire HAL, est destinée au dépôt et à la diffusion de documents scientifiques de niveau recherche, publiés ou non, émanant des établissements d'enseignement et de recherche français ou étrangers, des laboratoires publics ou privés. 


\title{
High-Frequency Mechanical Properties of Tumors Measured by Brillouin Light Scattering
}

\author{
Jérémie Margueritat, ${ }^{1}$ Angélique Virgone-Carlotta, ${ }^{1}$ Sylvain Monnier, ${ }^{1}$ Hélène Delanoë-Ayari, ${ }^{1}$ Hichem C. Mertani, ${ }^{2}$ \\ Alice Berthelot, ${ }^{1}$ Quentin Martinet, ${ }^{1}$ Xavier Dagany, ${ }^{1}$ Charlotte Rivière, ${ }^{1}$ Jean-Paul Rieu, ${ }^{1}$ and Thomas Dehoux ${ }^{1, *}$ \\ ${ }^{1}$ Institut Lumière Matière, UMR5306, Université Lyon 1-CNRS, Université de Lyon, 69622 Villeurbanne, France \\ ${ }^{2}$ Université de Lyon, Université Lyon 1, INSERM U1052, CNRS UMR 5286, \\ Centre de Recherche en Cancérologie de Lyon, Centre Léon Bérard, Lyon, France
}

(Received 7 June 2018; published 8 January 2019)

\begin{abstract}
The structure of tumors can be recapitulated as an elastic frame formed by the connected cytoskeletons of the cells invaded by interstitial and intracellular fluids. The low-frequency mechanics of this poroelastic system, dictated by the elastic skeleton only, control tumor growth, penetration of therapeutic agents, and invasiveness. The high-frequency mechanical properties containing the additional contribution of the internal fluids have also been posited to participate in tumor progression and drug resistance, but they remain largely unexplored. Here we use Brillouin light scattering to produce label-free images of tumor microtissues based on the high-frequency viscoelastic modulus as a contrast mechanism. In this regime, we demonstrate that the modulus discriminates between tissues with altered tumorigenic properties. Our micrometric maps also reveal that the modulus is heterogeneously altered across the tissue by drug therapy, revealing a lag of efficacy in the core of the tumor. Exploiting high-frequency poromechanics should advance present theories based on viscoelasticity and lead to integrated descriptions of tumor response to drugs.
\end{abstract}

DOI: 10.1103/PhysRevLett.122.018101

Tumors have a complex structure containing several cell types that are connected together by transmembrane bonds or extracellular matrix interactions. From a mechanical point of view, this structure can be recapitulated as an elastic frame formed by the connected cytoskeletons of the cells invaded by interstitial and intracellular fluids, with a behavior resembling that of a poroelastic material [Fig. 1(a)] $[1,2]$. In this system, the internal stresses built up by slow quasistatic external loading, during which the fluids are drained out, are borne only by the elastic skeleton [3]. The control of such a compression that occurs during tumor growth [4] has opened new therapeutic routes in which, for example, alleviating cell-cell adhesion strength in tumoral tissues with antibodies targeting cell adhesion proteins leads to a greater accumulation of therapeutic agents [5]. It has also been proposed that compressive stresses could trigger metastatic processes [6] and that monitoring intercellular adhesion strength could predict invasiveness [7].

This long-timescale response to strain can be investigated by observing shape relaxation [8] or cell-cell rearrangements using elastographic techniques $[9,10]$. Such measurements, usually interpreted as the response of a metastable system with multiple closely spaced relaxation times, have been recently revisited in light of poroelasticity, suggesting that the fluid phase also contributes to the total stress [11]. This internal fluid pressure, not probed by quasistatic experiments, has also been posited to participate in tumor progression and drug resistance [12]. By analogy with unconsolidated rock poromechanics [13], it is, on the other hand, expected that the response to a fast compression would contain the additional contribution of the biological fluids acting as if trapped in the porous elastic frame [14]. For this, it becomes crucial to probe the short-term mechanical response of tumors to explore the role of poroelasticity in response to drugs and to advance present theories based on viscoelasticity.

Moreover, although it is known from atomic force microscopy maps [15] that stiffness is not uniform in tumors, the quasistatic elastic modulus is usually assumed to be constant over the whole tissue to feed imaging algorithms. It is also evident from maps of the deformation field measured in spheroids by optical coherence tomography [16] that there is an anisotropic distribution of the strain within tissues, assumed to originate from a radial distribution of cell polarity and density [17]. An increased pressure has also been observed in the core of spheroids correlated to a slowing down of the proliferation rate [18]. Yet the true distribution of intrinsic mechanical properties was never characterized, hampering the description of internal tumor mechanics.

To circumvent present technical limitations, we implement a label-free imaging of the high-frequency poroelastic properties of tumor microtissues using Brillouin light scattering (BLS) microscopy. BLS has been used recently to image the mechanical properties of single cells using spectroscopic [19] and time-resolved implementations [20], 


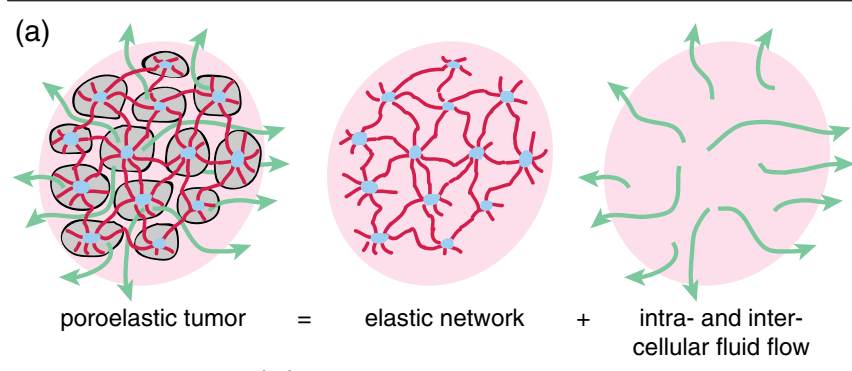

(a)

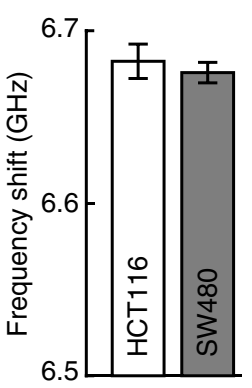

(b)

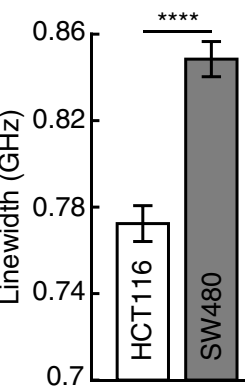

(c)

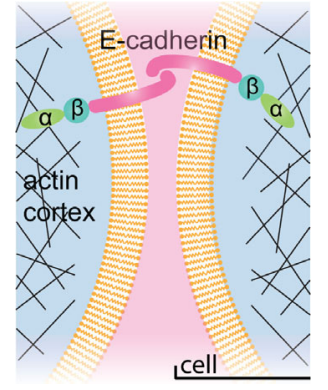

(b)

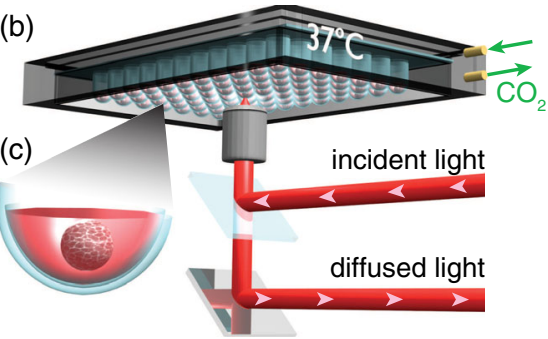

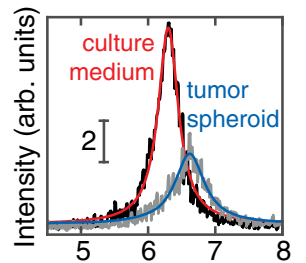

(d) (d) Frequency $(\mathrm{GHz})$

FIG. 1. In vitro Brillouin imaging setup. (a) Schematic of a poroelastic tumor. The cytoskeletons of each cell are connected together to form an elastic network. This network is invaded by intra- and intercellular fluids that are set in motion by acoustic vibrations. (b) Schematic of the setup. The laser beam is fed into a standard life science microscope. We equipped the motorized translation stage with a clip-on compact incubator that accepts standard 96-well plates and maintains physiological conditions at $37^{\circ} \mathrm{C}$ and $5 \% \mathrm{CO}_{2}$. The diffused light is collected and sent to the spectrometer. (c) Enlargement of a single well (the curvature of the round bottom has been exaggerated). Each well is filled with culture medium and contains one spheroid. (d) Typical spectra obtained in culture medium (black line) and tumor spheroid (gray line). Fits to Lorentzian functions are plotted in red and blue, respectively.

as well as on fixed ex vivo mouse brain tissues [21], cerebrospinal fluid [22], and cornea [23]. In BLS, the infinitesimal compressive motion induced by the spontaneous acoustic phonons creates a periodic grating of refractive index fluctuations. Using a monochromatic visible light beam, this grating with a spacing imposed by the acoustic wavelength produces a shift $f$ of the frequency of the backscattered light in the $\mathrm{GHz}$ range as exemplified by the spectrum obtained in culture medium in Fig. 1(d). The position and linewidth of the peak probe the longitudinal modulus at hypersonic frequencies. To image the mechanical properties of tissues, we coupled a scanning multipath Fabry-Perot interferometer with an inverted bioscience microscope (see Supplemental Material Note 1 [24]). The typical size of a cell within the spheroid is $\sim 5 \mu \mathrm{m}$. In order to observe a poroelastic unit element formed by several cells, we focused the probe light with a $20 \times$ objective lens (N.A. 0.35 ) to obtain an $\sim 10 \mu$ m laser spot diameter. We limited the axial spreading of the illuminated volume to $\sim 60 \mu \mathrm{m}$ using confocal pinholes (see Supplemental Material Note 3 [24]). Assuming that the

FIG. 2. Brillouin microscopy distinguishes colorectal spheroids with different metastatic properties. (a) Frequency shift and (b) linewidth measured from Brillouin spectra in HCT116 $(n=23)$ and SW480 $(n=27)$ spheroids $\left({ }^{* * *} p<0.0001\right.$, unpaired two-tailed $t$ test). (c) Schematic representation of a cell-cell contact. E-cadherins link the two cells through the intercellular space and bind to the intracellular actin cortex via the $\alpha$ - and $\beta$-catenins.

sample is homogeneous over this scattering volume, as in a Darcy-scale representation [27], the BLS spectra can be interpreted as the response of a damped harmonic oscillator with frequency position $f$ and full width at half maximum $\Gamma$. In this approximation, the storage modulus is $M^{\prime}=$ $\rho(f \lambda / 2 n)^{2}$, where $\rho$ is the mass density, $\lambda$ the laser wavelength, and $n$ the optical index. The low numerical aperture of the lens induces negligible broadening of the spectra $(\lesssim 2 \%)$ [21], allowing us to ascribe $\Gamma$ to the loss modulus $M^{\prime \prime}=\rho \Gamma f(\lambda / 2 n)^{2}$ [28]. To allow Brillouin investigation in physiological conditions, we used a transparent stage top incubator that maintains the samples in a $37^{\circ} \mathrm{C}$ and $5 \% \mathrm{CO}_{2}$ environment, while offering full optical access from the top and bottom sides for BLS and optical microscopy, respectively. The incubator is designed to accommodate standard 96-well plates for high-throughput probing and drug screening [Fig. 1(b)].

To demonstrate BLS on in vitro living tissues, we recreated the mechanical complexity of solid tumors with a reductionist approach. We used spheroids, a powerful in vitro model that is widely used in standardized preclinical studies to accelerate the translation of novel therapeutics to the clinic but also as a tool to understand precisely tumor physics and biology [29]. We engineered spheroids from the spontaneous aggregation of two different colorectal carcinoma cell lines, SW480 and HCT116 (see Supplemental Material Note 5 [24]) and placed each of them in an ultralow adhesion round-bottom well of a 96well plate [Fig. 1(c)]. The typical diameter of an untreated spheroid is $350 \mu \mathrm{m}$. We probed the Brillouin spectra at the center of the spheroids [see typical spectrum in Fig. 1(d)], and we extracted the Brillouin shift and linewidth [Figs. 2(a) and 2(b)]. No significant difference is visible on the shift, pointing to negligible differences in the refractive index $n$ and similar storage moduli $M^{\prime}$ (see Supplemental Material Note 9 [24]). On the other hand, the linewidth is clearly 
higher in SW480 spheroids, suggesting a higher loss modulus $M^{\prime \prime}$.

In our experiments, the loss modulus $M^{\prime \prime}$ describes the relaxation of structures smaller than the acoustic wavelength, $230 \mathrm{~nm}$ (see Supplemental Material Note 1 [24]) averaged over the scattering volume. At such a scale, we expect the dominant contribution of cell-cell adhesions of typical size $\gtrsim 180 \mathrm{~nm}$ (estimated from tight epithelial junctions) [30]. Interestingly, among the various molecular actors of mechanobiology, SW480 and HCT116 cell lines differ by genetic mutations of $\beta$-catenin and variations in the localization of $\alpha$-catenin. These proteins serve as a link between the transmembrane E-cadherins that join adjacent cells and the intracellular actin cortex, as illustrated in Fig. 2(c). Their alteration has been associated with a modified viscosity in mouse embryonic carcinoma F9 cell line in the low-frequency regime [31]. We observe a similar effect in the high-frequency loss modulus, suggesting the ability of BLS to discriminate between tumor tissues with different metastatic potential. In the future, further investigation of the link between cell-cell adhesion and Brillouin linewidth will allow deeper understanding of the mechanobiological underpinnings of metastasis.

We now focus on the in-depth distribution of these mechanical properties. We mapped the Brillouin spectra of untreated HCT116 spheroids with an $\sim 10 \mu \mathrm{m}$ step raster pattern in a quadrant of the equatorial plane [Fig. 3(a)]. At each pixel, the recorded spectrum showed a single Brillouin peak in the frequency range of interest. The image formed by $f$ [Fig. 3(b)] clearly reveals the gradient of storage modulus within the spheroid; the shift is larger in the center than on the edge, illustrating the increase of the storage modulus from the inner edge to the core (see Supplemental Material Note 1 [24]). The image formed by $\Gamma$ [Fig. 3(c)] shows higher values (more fluid) in a rim comprised between $\sim 150$ and $\sim 50 \mu \mathrm{m}$. We also observe regions with different optical contrasts in the bright field image [see red dotted line in Fig. 3(a)] that show up as well in the $f$ image Fig. 3(b). To verify these trends, we probed $f$ and $\Gamma$ at three positions (in the rim, in the intermediate region, and at the center) in $n=38$ spheroids. We confirm the largely constant $\Gamma$ [Fig. 3(e) suggesting constant $M^{\prime \prime}$ and $n$ values] and increasing $f$ from the rim to the center [Fig. 3(d)], pointing to an increase in $M^{\prime}$. From the intermediate region to the center, we observe opposite trends ( $f$ increases while $\Gamma$ decreases). Although we cannot access $n$ directly (see Supplemental Material Note 9 [24]), since both $M^{\prime}$ and $M^{\prime \prime}$ have a $1 / n^{2}$ dependence, this observation demonstrates that the mechanical properties are not homogeneously distributed within the spheroid.

To quantify these profiles, we plot the circular average of the images in Figs. 3(f) and 3(g) (see Supplemental Material Note 4 [24]). While the transition from the medium to the spheroid is clearly identified on the average frequency shift [see downward arrow in Fig. 3(f)], the average linewidth profile shows a steplike feature in the (a)

(b)
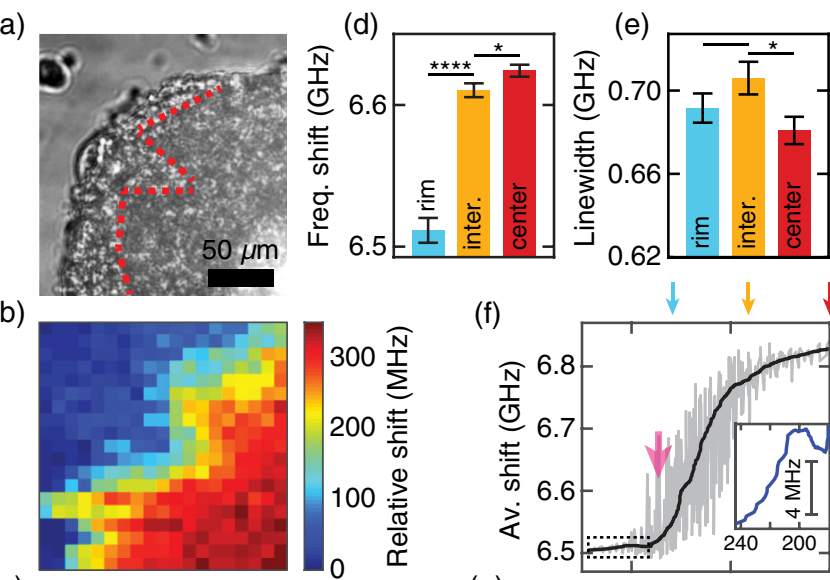

(c)

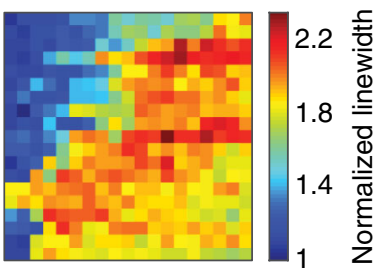

(f)

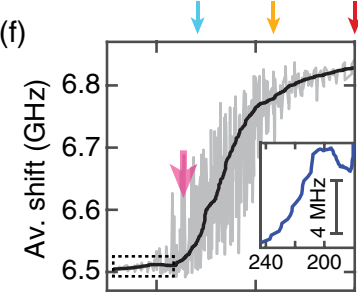

(g)

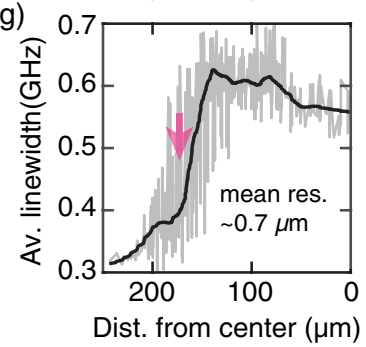

FIG. 3. Brillouin maps of the in-depth distribution of mechanical properties within the spheroid. (a) Phase contrast image of the untreated HCT116 spheroid (scale bar: $50 \mu \mathrm{m}$ ). As a guide to the eye, we separate with a red dotted line regions with different contrasts. (b),(c) Maps of the frequency shift $f$ and linewidth $\Gamma$. (d),(e) Frequency shift and linewidth measured at three positions [in the rim, in the intermediate region, and at the center; see arrows in Figs. 1(f) and 1(g)] in HCT116 spheroids ( $n=38,{ }^{* * * *} p<0.0001,{ }^{*} p<0.05$, unpaired two-tailed $t$ test). (f),(g) Circular average of the images in (b) and (c).

outer rim of the spheroid. An enlargement of the average frequency shift [inset of Fig. 3(f)] reveals a similar step with an $\sim 5 \mathrm{MHz}$ amplitude [32]. The steplike feature is correlated to the presence of a loose peripheral layer of cells observed by phase contrast microscopy [see, for instance, Fig. 4(a)] and suggests an exquisite sensitivity to escaping of cells in the surrounding environment. Importantly, these radial profiles quantify for the first time the in-depth distribution of mechanical properties in tumoral tissues.

Let us now test if such properties can be used to follow an anticancer therapy. We subjected the HCT116 spheroids to a two-day therapy with 5-fluorouracil (5-FU), a drug widely used in the treatment of colorectal cancers. 5-FU acts during the $S$ phase of the cell cycle and inhibits DNA synthesis by restricting availability of thymidylate and can also inhibit ribonucleic acid synthesis [33]. Although this drug has had impact on advanced tumors, its efficacy in first-line treatments remains limited due to tumor resistance [34]. We checked how the mechanical properties correlate with the kinetics of drug action. We formed spheroids, and 5-FU was then diluted in cell culture medium at a $300 \mu \mathrm{M}$ concentration. We checked drug efficacy at day 2 using a dead assay and phase contrast imaging; the spheroid diameter 

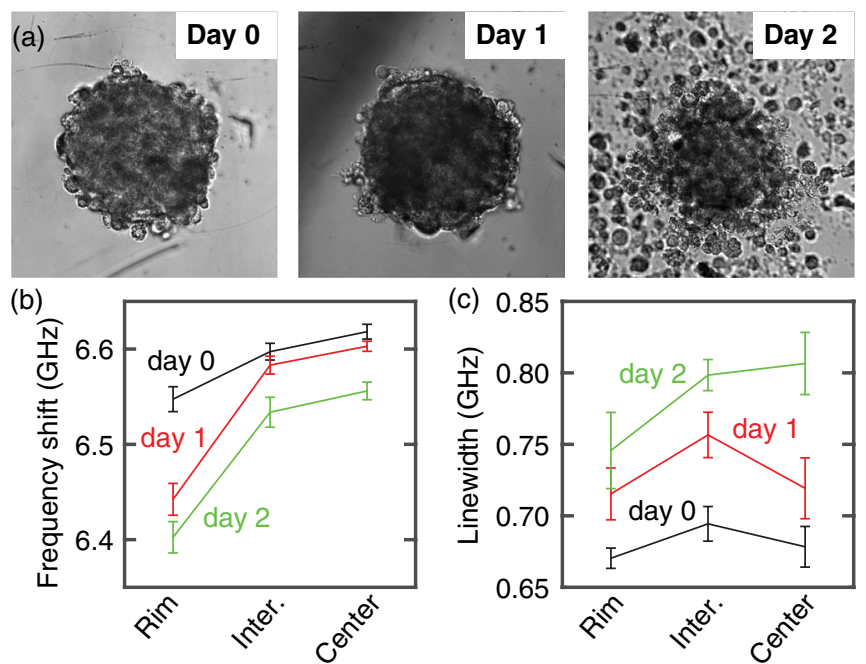

FIG. 4. Brillouin monitoring of two-day chemotherapy. (a) Optical images of 5-FU treated HCT116 spheroids at days 0 (beginning of the therapy), 1, and 2. At day 2, the spheroid is clearly disaggregated at the edges. (b) Frequency shift and (c) linewidth measured at three positions (in the rim, in the intermediate region, and at the center) at days 0,1 , and 2 .

decreases and the number of dead cells per $\mathrm{mm}^{3}$ increases, both confirming the action of the drug (see Supplemental Material Note 7 [24]) [35]. Each day from day 0 of the treatment, we also probed $f$ and $\Gamma$ at three positions [in the rim, in the intermediate region, and at the center, Figs. 4(b) and 4(c), respectively]. At day 0, we recognize the patterns we observed in Fig. 3. After one day of exposure, the storage modulus decreases significantly in the outer rim while remaining unaffected in the deeper regions of the tissue. Conversely, the loss modulus is increased largely homogeneously throughout the tissue. At day 2, stiffness is lowered in the whole spheroid, demonstrating its complete loss of cohesion upon drug action. Similarly, the spheroid is fluidized entirely, with a more pronounced effect at the center. Clearly, these results demonstrate the ability of BLS to quantify in-depth drug efficacy.

To further discuss light-scattering observations in the $\mathrm{GHz}$ range in tumors, the stress tensor $\sigma_{i j}$ and mean fluid pressure $p$ are estimated on the basis of a minimal Darcyscale poroelastic model [27] as a function of the strain tensor $\varepsilon_{i j}$ and fluid content $\xi$ written in index notation as

$$
\begin{gathered}
\sigma_{i j}=K_{u} \varepsilon_{k k} \delta_{i j}+2 \mu \varepsilon_{i j}-\frac{K_{u}-K_{d}}{\beta} \xi \delta_{i j}, \\
p=\frac{K_{u}-K_{d}}{\beta^{2}}\left(\xi-\beta \varepsilon_{k k}\right),
\end{gathered}
$$

where $K_{u}$ and $K_{d}$ are the undrained and drained moduli, respectively, $\mu$ is the shear modulus of the drained material (shear moduli of the intra- and intercellular fluids are neglected), and $\delta_{i j}$ is the Kronecker delta. The long-term stress-strain response of the tissue, classically observed in literature [1], simulates the drained case where the fluid is allowed to flow freely $(p=0)$ and gives access to $K_{d}$. In this limit, the Biot coefficient $\beta$ can be interpreted as the ratio $\xi / \varepsilon_{k k}$. Conversely, in the high-frequency limit, the fluid behaves as if trapped in the pores $(\xi=0)$ :

$$
\begin{gathered}
\sigma_{i j}=K_{u} \varepsilon_{k k} \delta_{i j}+2 \mu \varepsilon_{i j}, \\
p=-\frac{K_{u}-K_{d}}{\beta} \varepsilon_{k k} .
\end{gathered}
$$

In such a Darcy-scale description, the fluid flow through the pores of the spheroid is approximated to a Poiseuille flow, and the friction between the elastic frame and the fluid $F$ defines the frequency limit between these two regimes, $f_{c}=F / \rho_{f}$, where $\rho_{f} \sim 1100 \mathrm{~kg} / \mathrm{m}^{3}$ is the mass density of the internal fluids. Obviously, no values exist for $F$ in spheroids, but by analogy with hydrogels where $F \sim$ $4-200 \times 10^{12} \mathrm{~N} \mathrm{~s} / \mathrm{m}$, we expect $f_{c} \sim 4-200 \mathrm{GHz}$ [36]. Contrary to standard approaches that infer a drained modulus from a quasistatic deformation $[1,16]$, here we probe the low-frequency region of the undrained regime. As we approach $f_{c}$, variations in the longitudinal modulus can be interpreted as a variation in the volume fraction of circulating water $\phi$, and $M^{\prime} \approx M_{w}^{\prime} / \phi^{3}$, with $M_{w}^{\prime}$ the longitudinal modulus of the fluid phase [37]. In this frame, the increase in $M^{\prime}$ towards the center that we observed in untreated spheroids can be interpreted as a reduction in the amount of circulating water.

Another interpretation consists in considering that we are deeper in the undrained regime, above $f_{c}$, and that we probe the undrained longitudinal storage modulus $M^{\prime}=K_{u}+2 \mu$. We estimated $M^{\prime}=\rho(f \lambda / 2 n)$ from the $f$ values measured on Fig. 3(f), using standard values $\rho=$ $1100 \mathrm{~kg} / \mathrm{m}^{3}$ and $n=1.4$. We fed this $M^{\prime}$ function to a finite element solver to evaluate the equilibrium equation $\nabla \cdot \boldsymbol{\sigma}=0$ in such a functionally graded elastic sphere (see Supplemental Material Note 8 [24]). We found that the radial stress remains largely constant, while the volumetric strain decreases from the inner edge to the core. From a phenomenological prospective, this is identical to an increase in $\phi$ as described above and consistent with the lower proliferation and higher apoptosis rates observed in the center of spheroids [38]. Such reasoning concurs with the discussion on the contribution of water to the frequency shift in hydrated tissues [39]. From this discussion, it is clear that the determination of $f_{c}$ is critical to make more decisive interpretations, and reproducing Darcy's experiment will be the next step to quantify friction coefficient $F$ in these systems.

In the frame of the above discussion, the progressive decrease of the storage modulus $M^{\prime}$ during the anticancer therapy indicates a drug-induced permeable front that progresses towards the center of the spheroid. This observation is in line with recent observation of the progressive disaggregation of HCT116 spheroids using time-lapse 
microscopy during 5-FU therapy [35]. Interestingly, the loss modulus $M^{\prime \prime}$ increases from day 1 at the center, even though $M^{\prime}$ remains unaffected at this early stage of the treatment. We can hypothesize that a smaller dose is active at the center at the early stages and that it triggers metabolic activation, as observed in 2D models [40]. Our results have also pointed to an influence of catenins on $M^{\prime \prime}$; it would thus be interesting to investigate a possible upregulation of cell-cell contacts activated by low drug dose in the center of the spheroid.

In summary, Brillouin microscopy enables mapping the intrinsic longitudinal modulus of tumoral tissues without any fluorophores or tags. With this technique, we demonstrated that the ability to probe poromechanics close to the undrained limit allows us to discriminate the metastatic potential of tissues and monitor the in-depth action of standard therapeutic drugs. These observations pave the way to in-depth probing of drug efficacy using the highfrequency modulus as a quantitative indicator. Our results also revealed a strong heterogeneity in the high-frequency response of untreated tumors, contrary to what is usually assumed. We anticipate that this information on highfrequency 3D mechanics will set new grounds for the interpretation of the mechanobiology of cancer.

This work was partly supported by the Agence Nationale de la Recherche (Grant No. ANR-17-CE11-0010-01), the Région Auvergne Rhône-Alpes (SCUSI Grant No. 1700991901), and the Institut Thématique MultiOrganisme Cancer within the frame of the Programme Plan Cancer (ITMO, Grant No. PC201507). The authors thank Amandine Durand-Terrasson and Denis Ressnikoff from the Centre Imagerie Quantitative Lyon Est for their support for SPIM imaging.

*thomas.dehoux@univ-lyon1.fr

[1] T. Roose, P. A. Netti, L. L. Munn, Y. Boucher, and R. K. Jain, Microvasc. Res. 66, 204 (2003).

[2] D. Ambrosi, S. Pezzuto, D. Riccobelli, T. Stylianopoulos, and P. Ciarletta, J. Elast., 129107 (2017).

[3] H. T. Nia et al., Nat. Biomed. Eng. 1, 0004 (2016).

[4] G. Helmlinger, P. A. Netti, H. C. Lichtenbeld, R. J. Melder, and R. K. Jain, Nat. Biotechnol. 15, 778 (1997).

[5] S. K. Green, G. Francia, C. Isidoro, and R. S. Kerbel, Mol. Cancer Ther. 3, 149 (2004).

[6] K. Alessandri et al., Proc. Natl. Acad. Sci. U.S.A. 110, 14843 (2013).

[7] B. S. Winters, S. R. Shepard, and R. A. Foty, Int. J. Cancer 114, 371 (2005).

[8] G. Forgacs, R. A. Foty, Y. Shafrir, and M. S. Steinberg, Biophys. J. 74, 2227 (1998).

[9] P. Marmottant, A. Mgharbel, J. Käfer, B. Audren, J.-P. Rieu, J.-C. Vial, B. van der Sanden, A. F. M. Marée, F. Graner, and H. Delanoë-Ayari, Proc. Natl. Acad. Sci. U.S.A. 106, 17271 (2009).
[10] C.-E. Leroux, F. Bertillot, O. Thouvenin, and A.-C. Boccara, Biomed. Opt. Express 7, 4501 (2016).

[11] C. Giverso and L. Preziosi, Math. Med. Biol. 29, 181 (2012).

[12] R. K. Jain, J. Clin. Oncol. 31, 2205 (2013).

[13] E. Detournay and A.H.D. Cheng, Comprehensive Rock Engineering (Pergamon Press, New York, 1993), pp. 113-171.

[14] O. F. Zouani, T. Dehoux, M.-C. Durrieu, and B. Audoin, Soft Matter 10, 8737 (2014).

[15] M. Plodinec et al., Nat. Nanotechnol. 7, 757 (2012).

[16] C.-E. Leroux, J. Palmier, A. C. Boccara, G. Cappello, and S. Monnier, New J. Phys. 17, 073035 (2015).

[17] M. Delarue, J.-F. Joanny, F. Jülicher, and J. Prost, Interface Focus 4, 20140033 (2014).

[18] M. E. Dolega, M. Delarue, F. Ingremeau, J. Prost, A. Delon, and G. Cappello, Nat. Commun. 8, 14056 (2017).

[19] G. Scarcelli, W. J. Polacheck, H. T. Nia, K. Patel, A. J. Grodzinsky, R. D. Kamm, and S. H. Yun, Nat. Methods 12, 1132 (2015).

[20] T. Dehoux, M. Abi Ghanem, O. F. Zouani, J.-M. Rampnoux, Y. Guillet, S. Dilhaire, M.-C. Durrieu, and B. Audoin, Sci. Rep. 5, 8650 (2015).

[21] S. Mattana, S. Caponi, F. Tamagnini, D. Fioretto, and F. Palombo, J. Innov. Opt. Health Sci. 10, 1742001 (2017).

[22] Z. Steelman, Z. Meng, A. J. Traverso, and V. V. Yakovlev, J. Biophoton. 8, 408 (2015).

[23] G. Scarcelli and S. H. Yun, Nat. Photonics 2, 39 (2008).

[24] See Supplemental Material at http://link.aps.org/ supplemental/10.1103/PhysRevLett.122.018101 for details on the setup design and characteristics, cell culture and assays, and discussion on the influence of the refractive index which includes Refs. [25,26].

[25] C. Kittel, Introduction to Solid State Physics, 6th ed. (John Wiley \& Sons, New York, 1986).

[26] F. Yang, T. J. Grimsley, S. Che, G. A. Antonelli, H. J. Maris, and A. V. Nurmikko, J. Appl. Phys. 107, 103537 (2010).

[27] T. Bourbié, O. Coussy, and B. Zinszner, Acoustics of Porous Media (Technip, Paris, 2000).

[28] D. A. Pinnow, S. J. Candau, J. T. LaMacchia, and T. A. Litovitz, J. Acoust. Soc. Am. 43, 131 (1968).

[29] K. E. Foley, Nat. Methods 14, 559 (2017).

[30] C. Bertocchi et al., Nat. Cell Biol. 19, 28 (2017).

[31] T. V. Stirbat, A. Mgharbel, S. Bodennec, K. Ferri, H. C. Mertani, J.-P. Rieu, and H. Delanoë-Ayari, PLoS One 8, e52554 (2013).

[32] Such an increased frequency quantification arises from the radial average and this feature would otherwise not be visible on raw $f$ images.

[33] C. Focaccetti et al., PLoS One 10, e0115686 (2015).

[34] D. B. Longley, D. P. Harkin, and P. G. Johnston, Nat. Rev. Cancer 3, 330 (2003).

[35] A. Virgone-Carlotta, M. Lemasson, H. C. Mertani, J.-J. Diaz, S. Monnier, T. Dehoux, H. Delanöe-Ayari, C. Rivière, and J.-P. Rieu, PLoS One 12, e0188100 (2017).

[36] T. Tanaka, L. O. Hocker, and G. B. Benedek, J. Chem. Phys. 59, 5151 (1973). 
[37] P. Chiarelli, A. Lanatà, M. Carbone, and C. Domenici, J. Acoust. Soc. Am. 127, 1197 (2010).

[38] F. Montel, M. Delarue, J. Elgeti, D. Vignjevic, G. Cappello, and J. Prost, New J. Phys. 14, 055008 (2012).
[39] P.-J. Wu, I. V. Kabakova, J. W. Ruberti, J. M. Sherwood, I. E. Dunlop, C. Paterson, P. Trk, and D. R. Overby, Nat. Methods 15, 561 (2018).

[40] Z. Bash-Imam et al., Oncotarget 8, 46219 (2017). 